\title{
Correction to: When the progresses in neonatology lead to severe congenital nephron deficit: is there a pilot in the NICU?
}

\author{
Silvia lacobelli ${ }^{1,2} \cdot$ Jean-Pierre Guignard ${ }^{3}$
}

Published online: 10 January 2022

(c) The Author(s), under exclusive licence to International Pediatric Nephrology Association 2021

\section{Correction to: Pediatr Nephrol \\ https://doi.org/10.1007/s00467-021-05338-8}

Due to an unfortunate error during the processing of the article, Table 1 and Table 2 were missing in the first published version of this article. The publisher apologizes for this mistake. The missing Tables are displayed below. The original article was corrected, where the tables are now to be found.

Table 1 Neonatal KDIGO AKI definition

\begin{tabular}{lll}
\hline Stage & Serum creatinine $(\mathrm{mg} / \mathrm{dl})$ & Urine Output $(\mathrm{ml} / \mathrm{kg} / \mathrm{h})$ \\
\hline 1 & $\geq 0.3$ rise within $48 \mathrm{~h}$ or $\geq 1.5-1.9 \times$ rise from baseline* & $\leq 1$ for $24 \mathrm{~h}$ \\
& $\quad$ within 7 days & \\
2 & $\geq 2-2.9 \times$ rise from baseline & $\leq 0.5$ for $24 \mathrm{~h}$ \\
3 & $\geq 3 \times$ rise from baseline or $\geq 2.5$ or KRT initiation & $\leq 0.3$ for $24 \mathrm{~h}$ \\
\hline
\end{tabular}

*(previous lowest value)

$A K I$ acute kidney injury, $K R T$ kidney replacement therapy

The online version of the original article can be found at https:// doi.org/10.1007/s00467-021-05338-8

Silvia Iacobelli

silvia.iacobelli@chu-reunion.fr

1 Réanimation Néonatale et Pédiatrique, Néonatologie, CHU

La Réunion, Site Sud, Saint Pierre, France

2 Centre d'Études Périnatales de l'Océan Indien UR 7388, Université de la Réunion, Saint Pierre, France

3 Lausanne University Medical School, CHUV-1011 Lausanne, Switzerland 
Table 2 Recommendations for the kidney follow up of at-risk very premature infants ( $<32$ weeks of gestation), VLBWI and/or experienced AKI

Exclusive breastfeeding promotion

Prudent introduction of solid food

Target regular and balanced growth, avoid rapid catch-up growth

Family lifestyle education, avoidance of nephrotoxins

Blood pressure measurement *

Urinary analysis*

Proteinuria*

Prudent dietary pattern, physical activity, avoidance of smoking

Blood pressure, BMI, and urinalysis
When ? / How often

First 6 months of life

After regaining birth weight

At discharge / During follow-up visits

Before 1 year of age / Annual

Before 1 year of age / Annual

If additional risk factors */ Every 2 years

From childhood onwards

From 18 years onwards / Biannualy

*A baseline kidney ultrasound should be performed to detect small kidneys, asymmetry or structural abnormalities. Any abnormalities in kidney function or ultrasound should be followed-up by a pediatrician or pediatric nephrologist

$V L B W I$ very low birth weight infants, $A K I$ acute kidney injury, $C K D$ chronic kidney disease, $B M I$ body mass index

Publisher's note Springer Nature remains neutral with regard to jurisdictional claims in published maps and institutional affiliations. 